\title{
Role of a Digital Clinical Decision-Support System in General Practitioners' Management of COPD in Norway
}

\author{
Varun Kumar Vijayakumar,' \\ Tehmina Mustafa, ${ }^{1,2}$ \\ Bjarte Kjell Nore, ${ }^{3}$ \\ Kjell Yngvard Garatun-Tjeldstø, ${ }^{4}$ \\ Øystein Næss, ${ }^{5}$ Odd Erik Johansen, (iD) ${ }^{6}$ \\ Bernt Bøgvald Aarli, 2,7 \\ 'Centre for International Health, \\ Department of Global Public Health and \\ Primary Care, University of Bergen, \\ Bergen, Norway; ${ }^{2}$ Department of Thoracic \\ Medicine, Haukeland University Hospital, \\ Bergen, Norway; ${ }^{3}$ Bergen Legevakt, Bergen, \\ Norway; ${ }^{4}$ CodeLab, Bergen, Norway; \\ ${ }^{5}$ Boehringer Ingelheim, Oslo, Norway; \\ ${ }^{6}$ Department of Medical Research, Bærum \\ Hospital, Vestre Viken Hospital Trust, \\ Gjettum, Norway; ${ }^{7}$ Department of Clinical \\ Science, University of Bergen, Bergen, \\ Norway
}

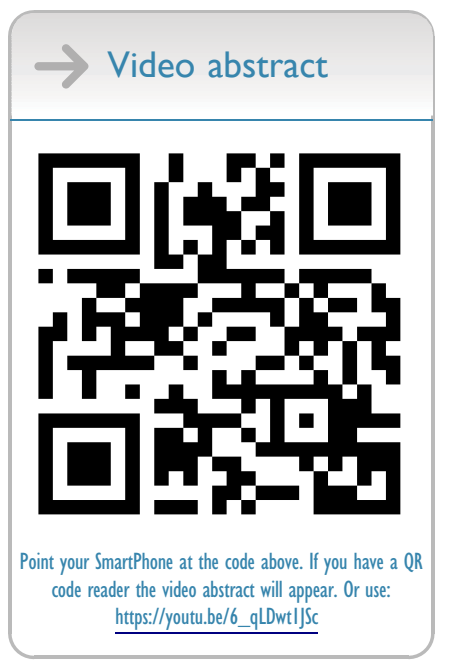

Correspondence: Bernt Bøgvald Aarli Department of Clinical Science, University of Bergen and Department of Thoracic Medicine, Haukeland University Hospital, Postboks I400, Bergen, 502I, Norway Tel +47 55 973-546

Email bernt.aarli@uib.no
Background: The study investigated if a web-based clinical decision-support system (CDSS) tool would improve general practitioners' (GPs) accuracy of diagnosis and classification of patients with chronic obstructive pulmonary disease (COPD), and whether nonpharmacological and pharmacological treatment would be better aligned with the COPD guidelines.

Methods: GPs were randomized to either a single use of the CDSS or continuing standard of care. The clinical recommendations of the CDSS were based on the GOLD guidelines and provided suggestions for treatment and management of COPD. Data were collected digitally from GPs and patients in both groups using a tablet computer. A follow-up questionnaire was sent to the GPs 1 year after the conclusion of the study.

Results: A total of 25 GPs (31\% women, mean age 41 years) participated, 12 randomized to using the CDSS tool and 13 followed standard of care when assessing their next five to ten COPD patients. In sum, 149 patients with presumed COPD were included (88 CDSS group, 61 standard-of-care group). In the CDSS group, no COPD misdiagnoses occurred, 98\% received vaccine recommendations, and all smokers $(n=39)$ received smoking-cessation advice. The standard-of-care group had 23\% misdiagnosis $(P<0.001)$, only $67 \%$ received vaccine recommendations $(P<0.001)$, and $87 \%$ smoking-cessation advice $(P=0.022$. All told, $31 \%$ of patients did not receive medication as recommended according to guidelines, with no significant differences between the groups. GPs rated the CDSS as very useful. Mean usage time was 3 minutes, 26 seconds. A majority (13 of 19, 68\%) of the GPs continued using the CDSS after the conclusion of the study. CAT score identified twice as many patients as having more symptoms than the mMRC, indicating the added value of the multi-item questionnaire.

Conclusion: Use of the CDSS was associated with preventing misdiagnosis of COPD and improved adherence to recommended nonpharmacological measures, but a single use did not improve pharmacological treatment considerations.

Keywords: COPD, GOLD, guidelines, decision support, CDSS

\section{Introduction}

Chronic obstructive pulmonary disease (COPD) is a preventable and modifiable condition characterized by irreversible or poorly reversible airflow obstruction and persistent respiratory symptoms due to airway and/or alveolar abnormalities. The disease is caused by prolonged exposure to noxious particles or gases, primarily from cigarette smoking. ${ }^{1}$ COPD contributes heavily to the morbidity and mortality rate worldwide. In 2019, it was the third-most common cause of all deaths 
globally, with over 3 million fatalities, ${ }^{2}$ accounting for $6 \%$ of all deaths. In a population-based study in Norway in people aged $>40$ years performed in 2015-2016, 6\% had COPD using the lower limit of normal of forced expiratory volume in 1 second $\left(\mathrm{FEV}_{1}\right)$ /forced vital capacity (FVC) ratio and the Global Lung Index $2012,{ }^{3}$ corresponding to 150,000 people. However, only half are currently receiving treatment for COPD according to the national prescription database, reflecting that a significant proportion are being undiagnosed, as is also seen in many other countries. ${ }^{4,5}$ This discrepancy could be related to several factors. One may be that general practitioners (GPs), the primary caregivers for patients with COPD, are overwhelmed by a rising number of different national, regional, and international guidelines across many different disease-areas for diagnostics, treatment, and followup, which increases the risk of information overload for physicians and risk of clinical inertia. ${ }^{6}$

As early diagnosis of COPD is recommended ${ }^{7}$ and establishing early-management strategies and initiating treatment has been seen to reduce both morbidity and mortality, ${ }^{8}$ tools to support GPs in identifying and managing people with COPD are needed, and a clinical decision-support system (CDSS) could be one.

CDSSs have various definitions. An early paper in clinical decision-making defined a CDSS as software that analyzes clinical information and presents conclusions (guidelines) for clinicians as output information. ${ }^{9}$ The input may be patient symptoms or results of lab or imaging investigations. Generated output may be diagnostic or therapeutic recommendations. Previous interventions with CDSSs have shown that if utilized successfully, such tools may increase adherence to evidence-based guidelines, reduce health-care costs, lead to a reduction in unnecessary diagnostic procedures being performed, and reduce inappropriate pharmacological treatment. ${ }^{10-12}$ CDSSs have been used in the diagnosis and management of chronic conditions such as hypertension, ${ }^{13}$ deep-vein thrombosis, ${ }^{14}$ asthma, ${ }^{15}$ and type 2 diabetes. ${ }^{16,17} \mathrm{~A}$ meta-analysis of a range of studies investigating the use of a CDSS on patients with asthma showed a positive impact of the intervention on management of the disease. ${ }^{18}$ At least two studies using CDSSs in managing acute exacerbation of COPD in the emergency department ${ }^{19,20}$ have been published, whereas there is a lack of studies investigating the impact of CDSSs at the primary-care level for managing stable COPD.
The present study explored the feasibility of an existing web-based CDSS tool for COPD in general practice. Our main goal was to investigate if such a tool would improve the accuracy of diagnosis and classification of COPD patients and whether nonpharmacological, and pharmacological treatment were aligned with COPD guidelines.

\section{Methods}

\section{Participants}

GP group practices with four or more doctors within a $16 \mathrm{~km}$ radius of Haukeland University Hospital, Bergen, Norway were invited personally in March 2019. Bergen has a population of approximately 275,000 and 238 GP practices. The GPs were randomized into two groups, one using an online digital CDSS for decision support, the other continuing to provide standard of care without the CDSS. Both groups were asked to include their next 5-10 patients with newly diagnosed or established COPD to participate in the study. A follow-up questionnaire was sent to the GPs 1 year after the conclusion of the study. Written informed consent was obtained from all patients and also from the GPs who completed the follow-up questionnaire. The study was approved by the Regional Committees for Medical and Health Research Ethics (REK 2018/947) in Norway and performed in accordance with the Declaration of Helsinki. A flowchart illustrating inclusion of patients and GPs for the study is shown in Figure 1.

\section{About the Digital CDSS}

The digital CDSS was based on the 2019 Global Initiative for Chronic Obstructive Lung Disease (GOLD) guidelines and the Norwegian COPD guidelines from 2012. The CDSS was developed by our group in cooperation with the Norwegian Heart and Lung Association. While the CDSS has been freely available for use since 2014, its use has not been widespread. For the purpose of the study, the existing web-based CDSS was customized to a "study version" for data-collection purposes. In addition, data were collected digitally using a tablet in both groups, transferring anonymized data to a secure study database. For the control arm, all information was collected on the tablet. 
INTERVENTION

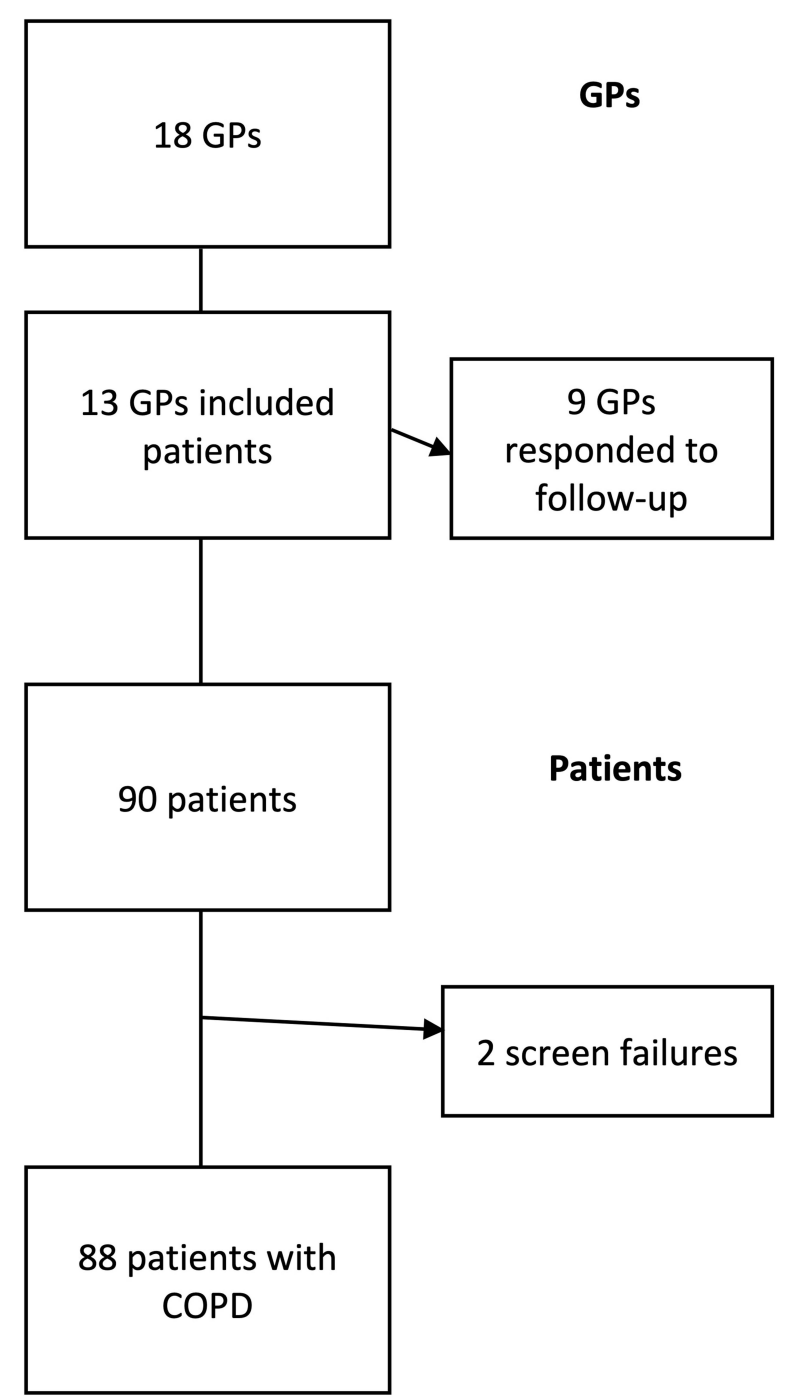

\section{CONTROL}

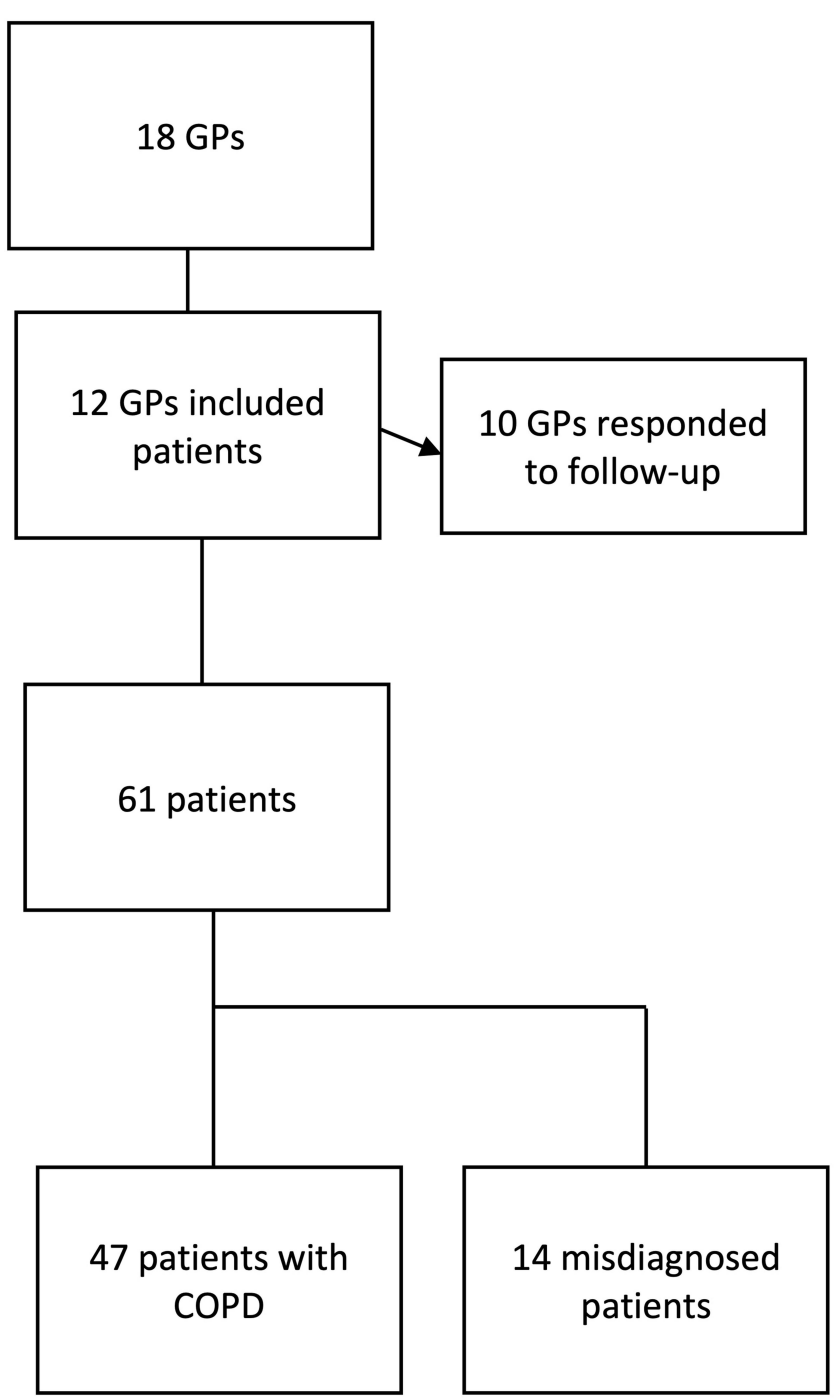

Figure I Flowchart illustrating inclusion of both general practitioners (GPs) and patients with chronic obstructive pulmonary disease (COPD) in the study. Feedback from the digital clinical decision-support system (CDSS) prevented inclusion of screen failures among GPs using the CDSS if spirometry did not show airway obstruction.

\section{Data Collection and System Feedback for GPs Using the CDSS}

GPs entered patients' sex, age, ethnicity, height, weight, smoking status, number of exacerbations requiring oral steroids and/or antibiotics or hospitalization the past year, modified Medical Research Council (mMRC) dyspneascale score, $^{21}$ and COPD-assessment (CAT) test score. ${ }^{22}$ During the clinic visit, all patients underwent spirometry and had $\mathrm{FEV}_{1}$ and FVC recorded.

The system-generated feedback to the GP included a summary of the results in a tabulated manner. If spirometry were consistent with airflow obstruction, defined as having an $\mathrm{FEV}_{1} / \mathrm{FVC}$ ratio $<0.7$ or below the lower limit of normal using the Global Lung Index $2012,^{3}$ a diagnosis of COPD was reported as "probable" under the condition that airflow obstruction was persistent. The severity of airflow limitation and the ABCD patient group according to the GOLD guidelines was also provided. ${ }^{1}$ If no obstruction was found on spirometry, the COPD diagnosis was labelled "unlikely", with spirometry feedback as either "normal" or as "restrictive pattern".

Treatment advice based on the GOLD ABCD group for each individual patient was provided, ie, first-line medication and additional medication suggestions in cases of symptoms of dyspnea or exacerbations. Finally, a summary of other COPD-management topics (smoking-cessation 
recommendations in smokers, physical exercise, pulmonary rehabilitation, and flu vaccination) was listed. At the end of the consultation, the GP handed over the tablet to the patients to complete the study questionnaires.

\section{Data-Collection Procedures for GPs Not Using the CDSS}

At the end of a COPD consultation, the GP filled out spirometry results and current medication used for COPD on the tablet and then handed it over to the patient to complete the study questionnaires.

\section{Patient-Reported Data}

The study questionnaires included mMRC dyspnea-scale and CAT scores, questions on exacerbation history, physical activity habits, and smoking status. All patients were asked if they had received information on physical activity, pulmonary rehabilitation, and/or flu vaccination during the consultation. Current smokers were asked if smoking cessation had been discussed.

\section{Follow-Up Data from GPs}

A follow-up questionnaire was sent to the GPs 1 year after conclusion of the study, where we asked for their age, sex, year of obtaining medical license, if they had used the CDSS following the completion of the study, and if that were the case, how useful they found it on a scale from $1-10$.

\section{Definition of Adherence to GOLD Guidelines}

Categorization in GOLD treatment groups was based on the degree of symptoms evaluated by both CAT and mMRC scores, in addition to exacerbation history. ${ }^{1}$ We defined appropriate medication as receiving medication as described by the GOLD ABCD medication group with/without addons for dyspnea and/or exacerbations, and "undertreated" if receiving less treatment. Patients were considered treated outside the GOLD guidelines if they were prescribed oral corticosteroids in stable COPD, inhaled corticosteroids (ICS) in a mono-inhaler, PDE4 inhibitor when $\mathrm{FEV}_{1}>50 \%$, two or more drugs belonging to the same medication class, if using both a short and a long-acting muscarinic antagonist, and if using montelukast for COPD.

\section{Definition of Misdiagnosis of COPD}

If no obstruction was found on spirometry, the diagnosis of COPD was considered misdiagnosed.

\section{Statistical Analysis}

Statistical analysis was performed using SPSS 26. Data are presented as means $\pm \mathrm{SD}$, medians and quartiles, or percentages. Independent-sample $t$-tests used for comparing data with normal distribution, while nonparametric data were compared with independent-sample Mann-Whitney $U$ and Chi-square tests.

\section{Results}

Figure 1 shows the study design. Of 36 GPs invited, 25 participated: 13 were randomized to use the CDSS (31\% women, mean age 41 years) and 12 to continue standard of care without the CDSS ( $33 \%$ women, mean age 50 years). In sum, 149 patients were included: 88 in the CDSS group (37 women, mean age 72 years) and 61 in the control group without the digital CDSS (30 women, mean age 68 years). Nineteen GPs (76\%) completed the follow-up questionnaire 1 year after the study.

Table 1 shows the characteristics of the GPs and patients in the intervention and control groups. Characteristics of the patients were mostly comparable. Only $77 \%$ in the control group had obstructive spirometry consistent with COPD. In patients without obstructive spirometry, eleven had normal spirometry, while three had lung volumes lower than the lower limit of normal, consistent with a restrictive spirometry (not shown in table). The COPD patients had mean $\mathrm{FEV}_{1}$ of $1.5 \mathrm{~L}(0.7)$ and FVC $2.8 \mathrm{~L}$ (1.0). Median CAT score was 13 (9). Characteristics of misdiagnosed patients were comparable for most COPD patients, differing only in spirometric results, with mean $\mathrm{FEV}_{1} 2.8 \mathrm{~L}$ (1.3) and FVC 3.1L (1.3; Table 2).

Nineteen GPs $(76 \%)$ completed the follow up questionnaire. Six in the non-CDSS group had misdiagnosed one or more patients. GPs who had included misdiagnosed patients were younger (mean age $35 \pm 7$ years), and had had their license to practice medicine for a shorter period (5.3 \pm 2.9 years) than GPs with no misdiagnosis: mean age 45 \pm 11 years, had had medical license $17 \pm 10$ years $(P=0.05)$.

\section{Symptom-Questionnaire Assessment}

A moderately strong positive correlation was found between mMRC and the CAT symptom scores $(r=0.47$, Figure 2). However, self-reported dyspnea on the mMRC 
Table I Characteristics of patients and GPs in the intervention and the control groups

\begin{tabular}{|c|c|c|c|}
\hline & Intervention & Control & $\boldsymbol{P}$ \\
\hline $\mathbf{n}$ & 88 & 61 & \\
\hline Age (years) & $72(9)$ & $68(9)$ & 0.014 \\
\hline Height (cm) & $168(9)$ & $|7|(9)$ & NS \\
\hline Women & $59 \%$ & $49 \%$ & NS \\
\hline Current smokers & $44 \%$ & $38 \%$ & NS \\
\hline FEV $_{\text {I (L) }}$ & I.5 (0.6) & $1.9(1.0)$ & NS \\
\hline FVC & $2.6(0.9)$ & $3.0(1.2)$ & NS \\
\hline FEV $/$ FVC & $0.57(0.11)$ & $0.61(0.19)$ & NS \\
\hline $\mathrm{mMRC}^{\mathrm{a}}$ & $I(I)$ & $I(I)$ & NS \\
\hline $\mathrm{CAT}^{\mathrm{a}}$ & $12(8)$ & $15(10)$ & 0.019 \\
\hline Exacerbations $^{\mathrm{a}}$ & $0(I)$ & $0(1)$ & NS \\
\hline Obstructive spirometry & $100 \%$ & $77 \%$ & $<0.001$ \\
\hline \multicolumn{4}{|l|}{ Drug class } \\
\hline SABA & $46(52 \%)$ & $40(66 \%)$ & NS \\
\hline SAMA & $4(5 \%)$ & $9(15 \%)$ & 0.030 \\
\hline LABA & $60(67 \%)$ & 44 (72\%) & NS \\
\hline LAMA & $65(73 \%)$ & $38(62 \%)$ & NS \\
\hline ICS & $46(52 \%)$ & 27 (44\%) & NS \\
\hline \multicolumn{4}{|l|}{ GP characteristics } \\
\hline$n^{b}$ & 9 & 10 & \\
\hline Age $(\text { years })^{b}$ & $49(12)$ & $39(9)$ & NS \\
\hline Women ${ }^{\mathrm{b}}$ & $33 \%$ & $40 \%$ & NS \\
\hline Clinical experience $^{b}$ & $17(12)$ & II (8) & 0.023 \\
\hline Number of patients ${ }^{b}$ & $8(4)$ & $5(1)$ & NS \\
\hline
\end{tabular}

Notes: Intervention group defined as GPs using the digital clinical decision-support system (CDSS). The control group continues standard care without the CDSS. Data presented as means (SD) unless otherwise, stated. ${ }^{a}$ Median (IQR). ${ }^{b}$ Only GPs who answered the follow-up questionnaire and provided signed consent.

Abbreviations: $F E V_{1}$, forced expiratory volume in I second; FVC, forced vital capacity; mMRC, modified Medical Research Council (dyspnea scale); CAT, COPDassessment test; SABA, short-acting beta 2 agonist; SAMA, short-acting muscarinic antagonist; LABA, long-acting beta 2 agonist; LAMA, long-acting muscarinic antagonist; ICS, inhaled corticosteroid.

questionnaire identified only 51 patients (34\%) as symptomatic (mMRC score $\geq 2$ ), while the composite CAT score (CAT $\geq 10)$, identified 110 patients $(73 \%)$ as symptomatic. Different proportions of patients in each $\mathrm{ABCD}$ treatment group were found on the CAT and mMRC (Figure 3). On the mMRC, $59 \%$ were defined as group A. With the CAT score, this group was reduced to $27 \%$, leaving group B as
Table 2 Comparison of characteristics between COPD and misdiagnosed patients

\begin{tabular}{|c|c|c|c|}
\hline & COPD & Misdiagnosed & $P$ \\
\hline Subjects (n) & 135 & 14 & \\
\hline Age (years) & 7I (9) & $67(11)$ & 0.014 \\
\hline Height (cm) & $169(9)$ & $169(10)$ & NS \\
\hline Women & $55 \%$ & $57 \%$ & NS \\
\hline Current smokers & $42 \%$ & $36 \%$ & NS \\
\hline FEV $_{\text {, (L) }}$ & $1.5(0.7)$ & $2.8(1.3)$ & $<0.001$ \\
\hline FEV ( (\% predicted) & $58(21)$ & $99(26)$ & $<0.001$ \\
\hline FVC (L) & $2.8(1.0)$ & $3.1(1.3)$ & NS \\
\hline FEV $/$ /FVC & $0.56(0.11)$ & $0.87(0.10)$ & $<0.001$ \\
\hline $\mathrm{mMRC}^{\mathrm{a}}$ & $I(I)$ & $I(I)$ & NS \\
\hline $\mathrm{CAT}^{\mathrm{a}}$ & $13(9)$ & $16(11)$ & NS \\
\hline Exacerbations $^{a}$ & $0(I)$ & $0(2)$ & NS \\
\hline GP characteristics & & & \\
\hline $\mathrm{n}$ & 13 & 6 & \\
\hline Age, years ${ }^{\mathrm{b}}$ & $45(\mathrm{II})$ & $35(7)$ & 0.004 \\
\hline Women ${ }^{\mathrm{b}}$ & $37 \%$ & $50 \%$ & NS \\
\hline Clinical experience $\dagger$ & $17(10)$ & $5.3(2.9)$ & $<0.001$ \\
\hline
\end{tabular}

Notes: Data presented as means \pm SD unless otherwise stated. ${ }^{a}$ Median (IQR). ${ }^{b}$ Only GPs who answered the follow-up questionnaire and provided signed consent. Abbreviations: $\mathrm{FEV}_{1}$, forced expiratory volume in I second; FVC, forced vital capacity; mMRC, modified Medical Research Council (dyspnea scale); CAT, COPDassessment test; GP, general practitioner.

the most represented $-55 \%$. On CAT scores, group $\mathrm{C}$ was almost eliminated.

\section{Pharmacological Treatment}

Pharmacological treatment with the various COPD medications is presented in Table 1. There were no notable differences in prescription patterns between the two groups, although patients in the control group were prescribed short-acting muscarinic antagonists (SAMAs) more often $(15 \%$ versus $5 \%$ ). Figure 4 shows how medication was prescribed in the medication groups. In group $\mathrm{A}$ and $\mathrm{B}$, some patients were not on any medication for COPD. Most patients in group $\mathrm{C}$ and $\mathrm{D}$ were on inhaled ICS. Almost a third (30\%) of the patients were incorrectly medicated according to GOLD guidelines, with no significant differences between the groups (Figure 5). Most 


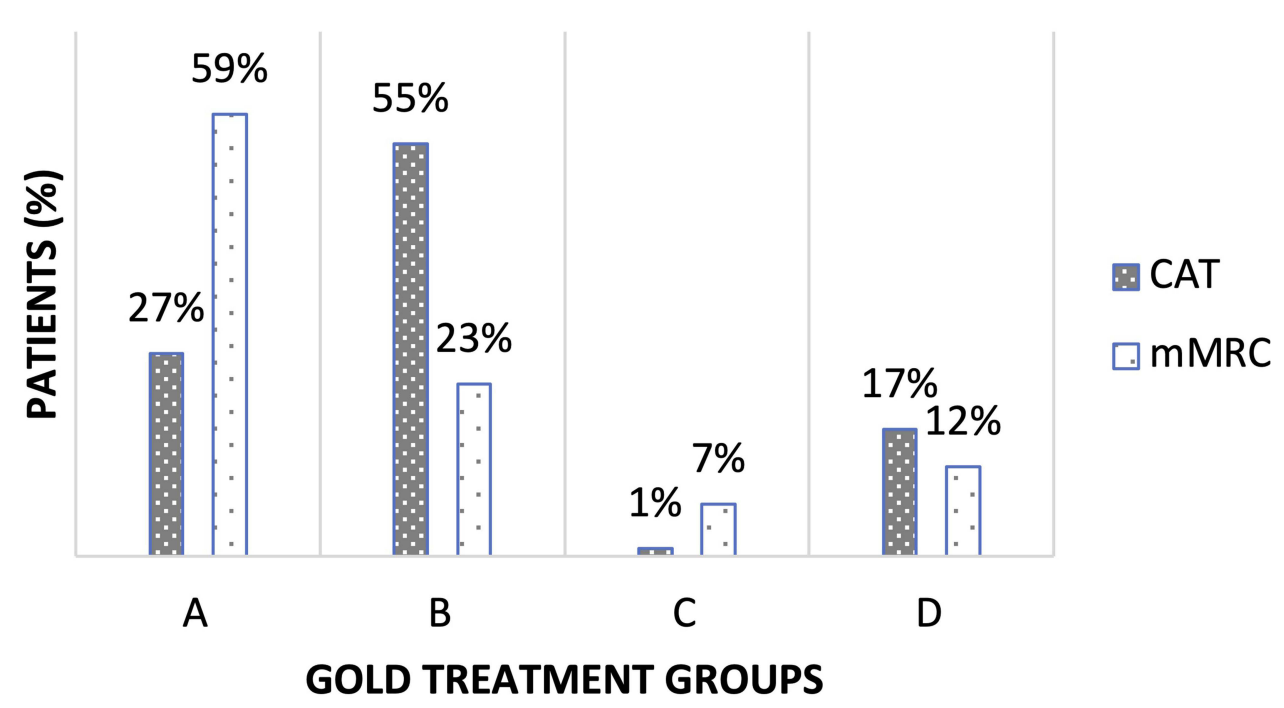

Figure 3 GOLD ABCD treatment groups in COPD patients defined by COPD-assessment test (CAT) score and bmodified Medical Research Council (mMRC) dyspneascale score, with misdiagnosed patients excluded $(n=135)$.

commonly, they were undertreated (17\%), receiving ICS in a mono-inhaler $(8 \%)$, or receiving two medications belonging to the same group of drugs (7\%). Two patients were on oral corticosteroids and an ICS mono-inhaler.

\section{Nonpharmacological Interventions}

A comparison of nonpharmacological interventions between the two groups is shown in Table 3. Significant differences between the groups were observed for flu vaccination and smoking-cessation recommendations. All current smokers in the intervention group were offered smoking-cessation advice and 98\% fluvaccination advice compared with $87 \%$ for smoking cessation and $67 \%$ for vaccine recommendations in the control group.

\section{User Satisfaction with the CDSS}

Mean time from first input until the GP reached the result page in the CDSS group was 3 minutes, 26 seconds. After the conclusion of the study, 13 of 19 (68\%) GPs were still using the CDSS, including several GPs in the control group. On the usefulness scale (1-10), the CDSS received a mean rating of $8.6 \pm 1.3$. Table 4 shows the characteristics of GPs who continued to use the digital CDSS after study conclusion. GPs not using the CDSS after study conclusion were significantly older and had had their medical license longer ( $P=0.05$, Table 4).
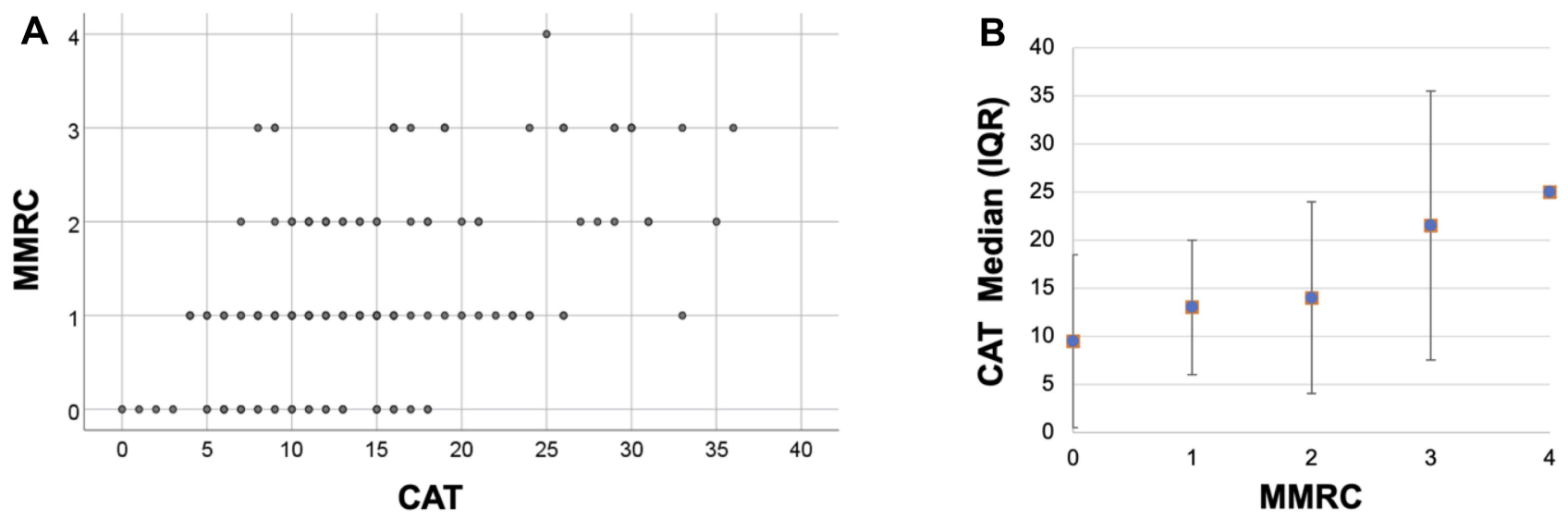

Figure 2 (A) Correlation between COPD-assessment test (CAT) and modified Medical Research Council (mMRC) dyspnea-scale scores ( $\mathrm{n}=\mathrm{I}$ 49, $r=0.47$; $P<0.00 \mathrm{I}$. (B) Distribution of CAT scores (median) according to mMRC score. Error bars represent the interquartile range. 


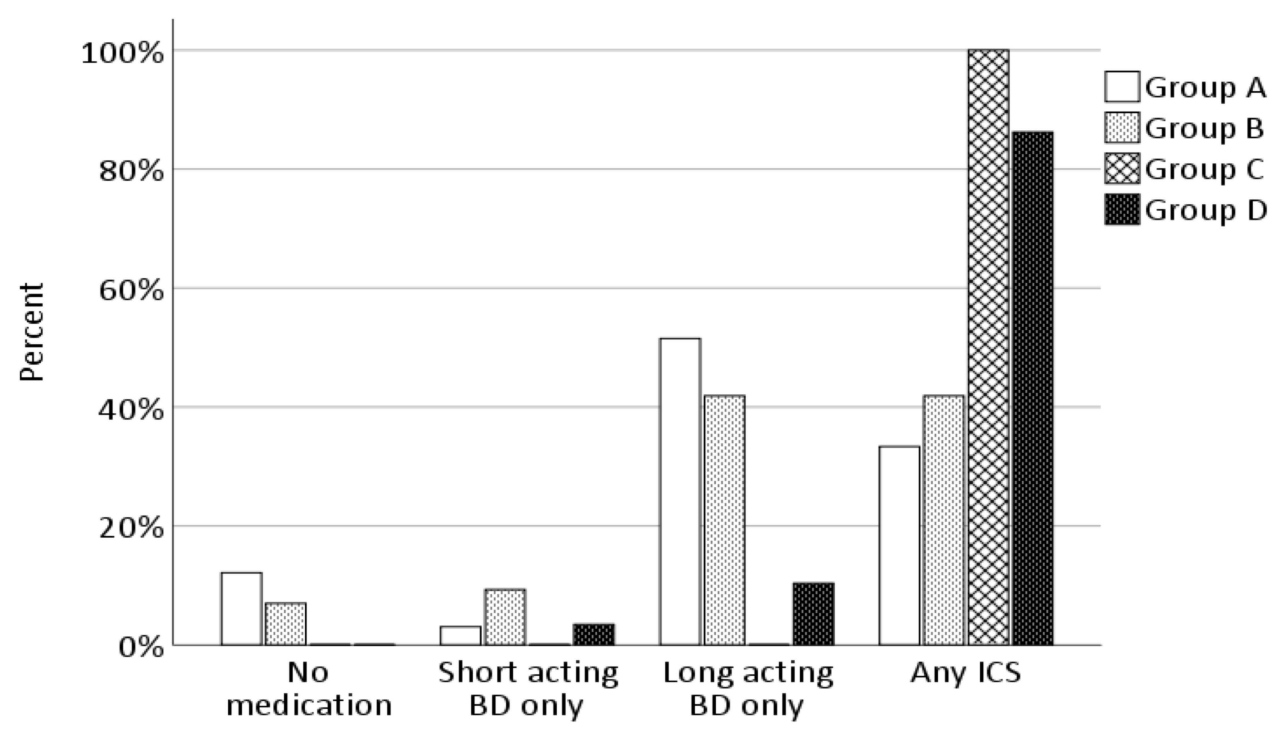

Figure 4 Medication use in the GOLD ABCD treatment groups generated using the COPD- assessment test for symptom evaluation. Abbreviations: BD, bronchodilator; ICS, inhaled corticosteroid.

\section{Discussion}

We investigated if a digital CDSS could increase GP adherence to guidelines for treating patients with COPD. The intervention prevented misdiagnosis and improved adherence to the nonpharmacological measures of smoking cessation and flu vaccination, but did not show a difference on pharmacological considerations used at a single visit.

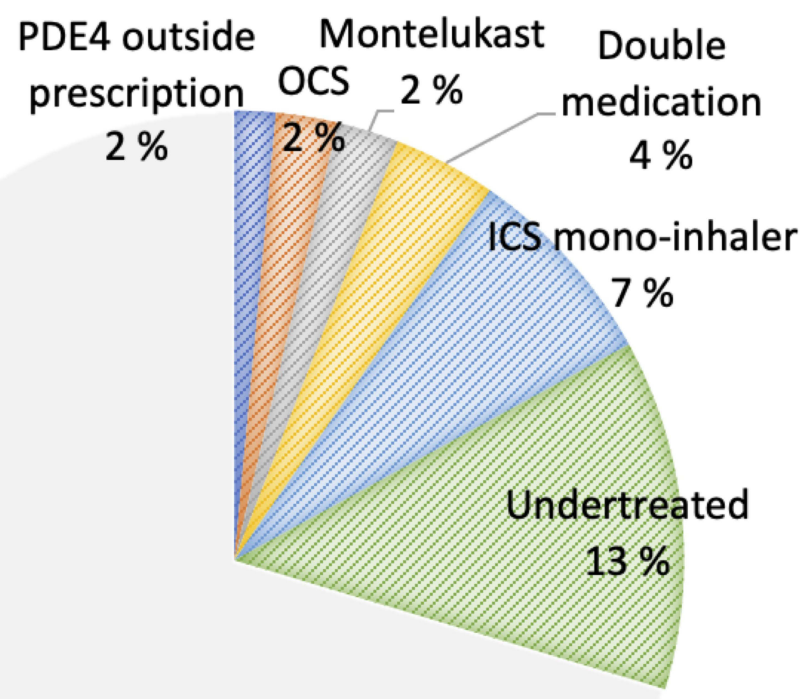

PDE4 outside prescription OCS

Montelukast

Double medication

a ICS mono-inhaler

Undertreated

Appropriate medication

\section{Appropriate medication \\ $70 \%$}

Figure 5 Pie chart showing medication among the patients. Appropriate medication defined as receiving medication as described by the GOLD ABCD medication group with/without add-ons for dyspnea and/or exacerbations.

Abbreviations: ICS, inhaled corticosteroid; PDE4, phosphodiesterase-4 inhibitor; OCS, oral corticosteroid. 
Table 3 Nonpharmacological treatment

\begin{tabular}{|l|c|c|c|}
\hline & $\begin{array}{c}\text { Intervention, } \\
\text { n (\%) }\end{array}$ & $\begin{array}{c}\text { Control, } \\
\text { n (\%) }\end{array}$ & P \\
\hline Smoking cessation $^{\mathrm{a}}$ & $39(100 \%)$ & $20(87 \%)$ & 0.022 \\
Physical activity & $73(82 \%)$ & $54(89 \%)$ & NS \\
Pulmonary rehabilitation & $13(15 \%)$ & $12(20 \%)$ & NS \\
Flu vaccination & $87(98 \%)$ & $41(67 \%)$ & $<0.00$ I \\
\hline
\end{tabular}

Notes: Intervention group defined as GPs using the digital clinical decision-support system (CDSS). The control group continued standard care without the digital CDSS. 'Among current smokers, there were 39 in the intervention group and 23 in the control group.

Table 4 Characteristics of GPs who continued using the digital CDSS after study conclusion

\begin{tabular}{|l|c|c|c|}
\hline & Continued use & No use & $\boldsymbol{P}$ \\
\hline Intervention $^{\mathrm{a}}$ & 5 & 4 & - \\
Control $^{\mathrm{a}}$ & 8 & 2 & - \\
Women $^{\mathrm{a}}$ & $54 \%$ & 0 & 0.024 \\
Age, years $^{\mathrm{a}}$ & $39(5)$ & $5 \mathrm{I}(14)$ & 0.026 \\
Clinical experience $^{\mathrm{a}}$ & $9(4)$ & $21(4)$ & 0.034 \\
\hline
\end{tabular}

Notes: Intervention group, GPs using the CDSS. Control group, GPs giving standard care without the digital CDSS. Data presented as means \pm SD. ${ }^{a}$ Only GPs who answered the follow-up questionnaire and provided signed consent.

The CDSS helped GPs in interpreting spirometry results and prevented misdiagnosis in the intervention group. We do not know the exact number of screen failures in the CDSS group, as very few GPs provided this information, but feedback from the CDSS prevented these patients from being included as COPD patients. Most patients who were misdiagnosed in the control group had completely normal spirometry, but shared a similar burden of respiratory symptoms to the COPD patients. This may have contributed to misdiagnosis. GPs with the shortest medical professional career had more COPD misdiagnoses, which may reflect better diagnostic skills with longer experience, although more seasoned GPs also simply may know their patients better.

The intervention did improve adherence to the nonpharmacological recommendations of smoking cessation and flu vaccination. Smoking cessation, vaccinations, physical activity, and pulmonary rehabilitation play important roles in the long-term management of the illness and treatment outcomes. In a busy everyday practice, these recommendations may be forgotten. Showing this information on the summary screen of the CDSS proved to be an effective reminder of nonpharmacological recommendations.
A third of the patients were either undertreated or received medication not recommended by the GOLD guidelines. There were no differences in pharmacological management between the groups, apart from less SAMA use in the intervention group. It is possible that the visual presentation on-screen was not perceived as important enough by the GPs to justify a change in medication or that the low number of participants made the study too underpowered to find such a difference. This might not have been a visit where the GP found it necessary to change medication. It is plausible that the lower prescription of SAMAs in the intervention group was due to treatment advice provided by the digital CDSS. If a patient had already received a long-acting muscarinic agonist (LAMA), a "stop" sign would appear on all SAMAs, indicating that no additional effect of a SAMA could be expected from this drug on top of a LAMA.

As a secondary objective, we investigated questionnaires used for symptom assessment. Very different proportions of patients were assigned in each $\mathrm{ABCD}$ treatment group when the mMRC scale was used compared with the CAT, and only half as many were defined as symptomatic on the former. The mMRC scale and CAT are considered equal in classifying COPD patients into $\mathrm{ABCD}$ treatment groups. The eight-item CAT identified twice as many patients in our study as having more symptoms than the mMRC scale. For this reason, we suggest adding a multi-item questionnaire when evaluating symptoms in patients who otherwise are defined as having a low grade of symptoms by mMRC-scale score alone $(<2)$.

Multiple studies have investigated adherence of GPs to COPD guidelines. There is no uniformity in the adherence of GPs to guidelines or recommendations. ${ }^{23}$ GP practices frequently fail to document lung function in COPD patients, ${ }^{24-}$ ${ }^{26}$ spirometry is often not performed adequately and may be interpreted incorrectly, ${ }^{27,28}$ and inappropriate medication is frequently prescribed. ${ }^{26,27}$ Such factors as lack of adequate knowledge and training and time constraints have been posited to be the major barriers. ${ }^{27}$ Educational programs aimed at improving GP practice in treating COPD patients do not seem to have a significant impact on diagnostic accuracy or pharmacological therapy. ${ }^{26}$

As all GPs in Norway use a computer and have an internet connection, a digital CDSS can easily be implemented for all clinicians. The digital CDSS was quite fast and received high marks on usefulness. Most of the GPs continued using it after the study. Those who did not were 
on average older and had more clinical experience. It could be that these physicians felt more confident in their clinical judgment. It is vital that the software is updated regularly to keep track of the latest changes in evidencebased guidelines and national recommendations. We also warn of a safety concern when using a secondary computer program in addition to a patient file system. When using two systems, there is always a risk that the information in one system does not match the same person in the other. If integrated into the patient file system, safety concerns regarding identity could be avoided and data could be retrieved from the patient file system, reducing input time.

\section{Limitations}

The CDSS has been available online since 2014. While we have usage statistics of the web page, it does not log user information, so we cannot say for certain if the GPs (in either arm) had used it before study start. GPs invited who chose not to participate may have induced a selection bias. However, the nonparticipation was random. We do not suspect the patient population to be very different between those who participated and those who did not. Likewise, the selection based on proximity to the hospital and the low number of GPs and patients participating in the study may have induced selection bias and limit the generalizability of the study. A longitudinal design would have been better suited to capture changes in medication, optimally with a duration of at least 12 months, which is the maximum duration of a prescription in Norway. The crosssectional design, examining the patients at a single point in time only, was chosen for cost and simplicity.

\section{Conclusion}

A digital CDSS tool prevented misdiagnosis of COPD in general practice and improved adherence to nonpharmacological interventions of flu vaccination and smoking cessation. The intervention did not influence pharmacological treatment choices. CAT scores identified twice as many symptomatic patients than mMRC dyspnea-scale scores, indicating that a multi-item questionnaire should be added when evaluating symptoms in patients who otherwise are defined as having a low degree of symptoms by mMRC score alone.

\section{Acknowledgments}

We would like to thank all GPs and patients who participated in the study and Boehringer-Ingelheim for funding, including iPADs used.

\section{Disclosure}

Bjarte Kjell Nore reports grants from Boehringer Ingelheim during the conduct of the study and personal fees from Boehringer Ingelheim, Novartis, and AstraZeneca outside the submitted work. Øystein Næss is employed by Boehringer Ingelheim Norway. Odd Erik Johansen reports personal fees and being an employee of Nestlé Health Science and a former employee of Boehringer Ingelheim outside the submitted work. Bernt Bøgvald Aarli reports grants from Boehringer Ingelheim during the conduct of the study and personal fees from Boehringer Ingelheim, AstraZeneca, Sanofi-Aventis Norway, Chiesi, Boehringer Ingelheim, and Novartis Norway, outside the submitted work. The authors report no other potential conflicts of interest for this work.

\section{References}

1. Vogelmeier C. The global strategy for diagnosis, management and prevention of COPD 2021 UPDATE. GOLD science committee members (2020-2021). 2020 [cited 2018; Available from: http://gold copd.org. Accessed July 27, 2021.

2. Global Health Estimates 2020. Deaths by Cause, Age, Sex, by Country and by Region, 2000-2019. Geneva: World Health Organization; 2020. Available from: https://www.who.int/newsroom/fact-sheets/detail/the-top-10-causes-of-death. Accessed July 27, 2021.

3. Nafstad P. Chronic Obstructive Pulmonary Disease (COPD) in Norway February 21, 2018 [cited January 1, 2021]; Available from: https://www.fhi.no/en/op/hin/health-disease/copd/. Accessed July 27, 2021.

4. Bednarek M, Maciejewski J, Wozniak M, et al. Prevalence, severity and underdiagnosis of COPD in the primary care setting. Thorax. 2008;63(5):402-407. doi:10.1136/thx.2007.085456

5. Quach A, Giovannelli J, Chérot-Kornobis N, et al. Prevalence and underdiagnosis of airway obstruction among middle-aged adults in northern France: the ELISABET study 2011-2013. Respir Med. 2015;109(12):1553-1561. doi:10.1016/j.rmed.2015.10.012

6. Brean A. Chaos. Tidsskr nor Laegeforen. 2016;136(8):687. doi:10.4045/tidsskr.16.0394

7. Csikesz NG, Gartman EJ. New developments in the assessment of COPD: early diagnosis is key. Int J Chron Obstruct Pulmon Dis. 2014;9:277-286.

8. Soriano JB, Zielinski J, Price D. Screening for and early detection of chronic obstructive pulmonary disease. Lancet. 2009;374 (9691):721-732. doi:10.1016/S0140-6736(09)61290-3

9. Owczarek A, Smertka M, Jędrusik P, et al. Computerized systems supporting clinical decision in medicine. Stud Log Gramm Rhetor. 2018;56(1):107-120. doi:10.2478/slgr-2018-0044

10. Monteiro L, Maricoto T, Solha IS, Monteiro-Soares M, Martins C. Computerised decision to reduce inappropriate medication in the elderly: a systematic review with meta-analysis protocol. $B M J$ Open. 2018;8(1):e018988.

11. Kawamoto K, Houlihan CA, Balas EA, et al. Improving clinical practice using clinical decision support systems: a systematic review of trials to identify features critical to success. BMJ. 2005;330 (7494):765.

12. Shahsavarani AM, Azad Marz Abadi E, Hakimi KM, Jafari S, Qaranli S. Clinical decision support systems (CDSSs): state of the art review of literature. Int J Med Rev. 2015;2(4):299-308. 
13. Anchala R, Kaptoge S, Pant H, et al. Evaluation of effectiveness and cost-effectiveness of a clinical decision support system in managing hypertension in resource constrained primary health care settings: results from a cluster randomized trial. J Am Heart Assoc. 2015;4 (1):e001213. doi:10.1161/JAHA.114.001213

14. Haut ER, Lau BD, Kraenzlin FS, et al. Improved prophylaxis and decreased rates of preventable harm with the use of a mandatory computerized clinical decision support tool for prophylaxis for venous thromboembolism in trauma. Arch Surg. 2012;147 (10):901-907. doi:10.1001/archsurg.2012.2024

15. Carroll AE, Anand V, Dugan TM, et al. Increased physician diagnosis of asthma with the child health improvement through computer automation decision support system. Pediatr Allergy Immunol Pulmonol. 2012;25(3):168-171. doi:10.1089/ped.2012.0143

16. Ampudia-Blasco FJ, Benhamou PY, Charpentier G, et al. A decision support tool for appropriate glucose-lowering therapy in patients with type 2 diabetes. Diabetes Technol Ther. 2015;17(3):194-202. doi:10.1089/dia.2014.0260

17. Ceriello A, Gallo M, Candido R, et al. Personalized therapy algorithms for type 2 diabetes: a phenotype-based approach. Pharmgenomics Pers Med. 2014;7:129-136.

18. Fathima M, Peiris D, Naik-Panvelkar P, et al. Effectiveness of computerized clinical decision support systems for asthma and chronic obstructive pulmonary disease in primary care: a systematic review. BMC Pulm Med. 2014;14(1):189. doi:10.1186/1471-2466-14-189

19. Epstein D, Barak-Corren Y, Isenberg Y, et al. Clinical decision support system: a pragmatic tool to improve acute exacerbation of COPD discharge recommendations. COPD. 2019;16(1):18-24. doi:10.1080/ 15412555.2019.1593342

20. Parikh R, Shah TG, Tandon R. COPD exacerbation care bundle improves standard of care, length of stay, and readmission rates. Int J Chron Obstruct Pulmon Dis. 2016;11:577. doi:10.2147/COPD. S100401
21. Fletcher CM. Standardised questionnaire on respiratory symptoms: a statement prepared and approved by the MRC committee on the aetiology of chronic bronchitis (MRC breathlessness score). BMJ. 1960;2:1665.

22. Jones PW, Harding G, Berry P, et al. Development and first validation of the COPD assessment test. Eur Respir J. 2009;34(3):648-654. doi:10.1183/09031936.00102509

23. Sehl J, O’Doherty J, O’Connor R, et al. Adherence to COPD management guidelines in general practice? A review of the literature. $\mathrm{Ir}$ J Med Sci. 2018;187(2):403-407. doi:10.1007/s11845-017-1651-7

24. Belletti D, Liu J, Zacker C, et al. Results of the CAPPS: COPD assessment of practice in Primary Care Study. Curr Med Res Opin. 2013;29(8):957-966. doi:10.1185/03007995.2013.803957

25. Kaufmann C, Markun S, Hasler S, et al. Performance measures in the management of chronic obstructive pulmonary disease in primary care - a retrospective analysis. Praxis. 2015;104(17):897-907. doi:10.1024/1661-8157/a002101

26. Bertella E, Zadra A, Vitacca M. COPD management in primary care: is an educational plan for GPs useful? Multidiscip Respir Med. 2013;8(1):1-7. doi:10.4081/mrm.2013.500

27. Perez X, Wisnivesky JP, Lurslurchachai L, et al. Barriers to adherence to COPD guidelines among primary care providers. Respir Med. 2012;106(3):374-381. doi:10.1016/j.rmed.2011.09.010

28. Davis KJ, Landis SH, Oh YM, et al. Continuing to confront COPD international physician survey: physician knowledge and application of COPD management guidelines in 12 countries. Int J Chron Obstruct Pulmon Dis. 2015;10:39.

\section{Publish your work in this journal}

The International Journal of COPD is an international, peer-reviewed journal of therapeutics and pharmacology focusing on concise rapid reporting of clinical studies and reviews in COPD. Special focus is given to the pathophysiological processes underlying the disease, intervention programs, patient focused education, and self management protocols. This journal is indexed on PubMed Central, MedLine and CAS. The manuscript management system is completely online and includes a very quick and fair peer-review system, which is all easy to use. Visit http://www.dovepress.com/testimonials.php to read real quotes from published authors. 